\title{
Moments of inertia for neutron and strange stars: Limits derived for the Crab pulsar
}

\author{
M. Bejger and P. Haensel
}

\author{
N. Copernicus Astronomical Center, Polish Academy of Sciences, Bartycka 18, 00-716 Warszawa, Poland \\ e-mail: haensel@camk.edu.pl
}

Received 28 March 2002 / Accepted 1 October 2002

\begin{abstract}
Recent estimates of the properties of the Crab nebula are used to derive constraints on the moment of inertia, mass and radius of the pulsar. To this purpose, we employ an approximate formula combining these three parameters. Our "empirical formula" $I \simeq a(x) M R^{2}$, where $x=\left(M / M_{\odot}\right)(\mathrm{km} / R)$, is based on numerical results obtained for thirty theoretical equations of state of dense matter. The functions $a(x)$ for neutron stars and strange stars are qualitatively different. For neutron stars $a_{\mathrm{NS}}(x)=x /(0.1+2 x)$ for $x \leq 0.1$ (valid for $\left.M>0.2 M_{\odot}\right)$ and $a_{\mathrm{NS}}(x)=\frac{2}{9}(1+5 x)$ for $x>0.1$. For strange stars $a_{\mathrm{SS}}(x)=\frac{2}{5}(1+x)$ (not valid for strange stars with crust and $M<0.1 M_{\odot}$ ). We obtain also an approximate expression for the maximum moment of inertia $I_{\max , 45} \simeq\left(-0.37+7.12 \cdot x_{\max }\right)\left(M_{\max } / M_{\odot}\right)\left(R_{M_{\max }} / 10 \mathrm{~km}\right)^{2}$, where $I_{45}=I / 10^{45} \mathrm{~g} \cdot \mathrm{cm}^{2}$, valid for both neutron stars and strange stars. Applying our formulae to the evaluated values of $I_{\text {Crab }}$, we derive constraints on the mass and radius of the pulsar. A very conservative evaluation of the expanding nebula mass, $M_{\text {neb }}=2 M_{\odot}$, yields $M_{\text {Crab }}>1.2 M_{\odot}$ and $R_{\text {Crab }}=10-14 \mathrm{~km}$. Setting the most recent evaluation ("central value") $M_{\text {neb }}=4.6 M_{\odot}$ rules out most of the existing equations of state, leaving only the stiffest ones: $M_{\text {Crab }}>1.9 M_{\odot}, R_{\text {Crab }}=14-15 \mathrm{~km}$.
\end{abstract}

Key words. dense matter - equation of state - stars: neutron

\section{Introduction}

The moment of inertia of neutron stars plays a crucial role in the models of radio pulsars. In the standard case of rigid rotation the total energy expenditure per unit time $\dot{E}_{\text {tot }}$ is related to the measured pulsar angular frequency $\Omega$ and its time derivative $\dot{\Omega}$ by $\dot{E}_{\text {tot }}=-I \Omega \dot{\Omega}$. However, even in the simplest case of slow rotation $\left(\Omega^{2} \ll(c / R)^{2} G M / R c^{2}, R \Omega \ll c\right)$ characteristic of observed radio pulsars, the relation of $I$ to the matter distribution within the star is complicated by the general relativistic effects such as the dragging of the local inertial frames. Among all global neutron star parameters the moment of inertia is the most sensitive to the dense matter equation of state (EOS). The "theoretical maximum mass" of neutron stars increases by a factor of two when going from the softest to the stiffest EOS. The "theoretical maximum moment of inertia" however increases then by a factor of seven (Haensel 1990). In view of this particular sensitivity of $I$ to the largely unknown equation of state (EOS) of dense matter at supra-nuclear density, it is of interest to look for observational evaluations $I$, which could then be used to constrain theoretical models.

In the present paper we use recent evaluations of the parameters of the expanding Crab nebula, in order to derive constraints on the Crab-pulsar neutron star. In Sect. 2 we reconsider the energetics of the Crab nebula, and using expandingshell model of the nebula we derive a constraint on the moment

Send offprint requests to: M. Bejger, e-mail: bejger@camk. edu.pl of inertia of the Crab pulsar. In Sect. 3 we derive approximate formulae for $I$ of neutron stars and strange stars, based on a statistical analysis of a numerical data sample obtained for thirty theoretical EOSs of dense matter. These formulae are then used in Sect. 4 to derive constraints in the $M-R$ plane, implied by the value of $I_{\mathrm{Crab}}$, deduced in Sect. 2. Appendix presents results of an analysis of the correlation between the maximum value of $I$ and the mass and radius of the configuration with maximum allowable mass.

\section{Energetics of the Crab nebula and constraints on $I_{\text {Crab }}$}

The present values of $\Omega$ and $\dot{\Omega}$ for the Crab pulsar are $188.119 \mathrm{~s}^{-1}$ and $-2.366 \times 10^{-13} \mathrm{~s}^{-2}$, respectively (Lyne \& Graham-Smith 1998). Therefore, the present rate of the rotational energy loss is

$I \Omega \dot{\Omega}=\dot{E}_{\text {rot }}=4.45 \times 10^{38} I_{45} \mathrm{erg} \mathrm{s}^{-1}$.

It is commonly accepted that $\dot{E}_{\text {rot }}$ has to account for the luminosity of the pulsar and the nebula, as well as for the accelerated expansion of the nebula, so that

$\dot{E}_{\text {rot }}>\dot{E}_{\text {rad }}+\dot{E}_{\text {exp }}$

Although the nebula has a complicated shape we will assume for simplicity that it is spherically symmetric with a radius 
$R_{\text {neb }} \simeq 1.25$ pc (see e.g. Douvion et al. 2001; Petersen 1998). The total power of radiation in the $\mathrm{X}, \mathrm{UV}$, and optical domains is given by Petersen (1998):

$\dot{E}_{\mathrm{rad}} \simeq 1.5 \times 10^{38}\left(\frac{d}{2 \mathrm{kpc}}\right)^{2} \mathrm{erg} \mathrm{s}^{-1}$.

Nugent (1998) calculated the velocities of expansion of 50 bright filaments by comparing their positions on four high-resolution photographs taken in 1939, 1960, 1976 and 1992. After projecting the motion of filaments backward in time, he obtained convergence in $\mathrm{AD} 1130 \pm 16 \mathrm{yr}$, in accordance with Trimble (1968). The calculated moment of the supernova event is by $\Delta=76 \mathrm{yr}$ later than the date recorded by the Chinese astronomers, AD 1054. Therefore, the actual expansion had a non-zero acceleration. Assuming a constant value of the acceleration, $\dot{v}$, and putting the present mean expansion velocity, determined from the measurements of the spectra, $v \sim 1.5 \times 10^{8} \mathrm{~cm} \mathrm{~s}^{-1}$ (e.g. Davidson \& Fesen 1985; Bietenholtz et al. 1991; Sollerman et al. 2000), we obtain

$\dot{v}=\frac{2 v \Delta}{T^{2}} \simeq 0.82 \times 10^{-3} \mathrm{~cm} \mathrm{~s}^{-2}$.

The Crab nebula expands in the interstellar, mostly hydrogenic, gas. Let the number density of the hydrogen atoms be $n_{\mathrm{H}}$. The expanding shell sweeps the interstellar medium, which becomes a part of the shell after being accelerated to the shell velocity. Consequently, the mass of the nebula increases at the rate

$\dot{M}_{\text {neb }}=4 \pi R_{\text {neb }}^{2} n_{\mathrm{H}} m_{\mathrm{H}} v$,

where $m_{\mathrm{H}}$ is the mass of the hydrogen atom. The corresponding rate of the kinetic energy injection needed to support the expansion is $\dot{M}_{\mathrm{neb}} v^{2} / 2$. The total power needed to support the nebula expansion is therefore

$\dot{E}_{\text {exp }}=M_{\text {neb }} v \dot{v}+2 \pi R_{\text {neb }}^{2} n_{\mathrm{H}} m_{\mathrm{H}} v^{3}$.

Under our assumptions, $\dot{E}_{\text {rot }}$ should exceed the sum $\dot{E}_{\text {rad }}+\dot{E}_{\text {exp }}$. This condition results in a lower-bound on the moment of inertia of the Crab pulsar,

$I_{\text {Crab }}>\frac{\dot{E}_{\text {exp }}+\dot{E}_{\text {rad }}}{\Omega|\dot{\Omega}|}$

which can be rewritten as

$$
\begin{aligned}
I_{\text {Crab }, 45}>0.34\left(\frac{d}{2 \mathrm{kpc}}\right)^{2}+0.55 \frac{M_{\text {neb }}}{M_{\odot}} \\
+0.15\left(\frac{R_{\text {neb }}}{1 \mathrm{pc}}\right)^{2} \frac{n_{\mathrm{H}}}{0.2 \mathrm{~cm}^{-3}} .
\end{aligned}
$$

We will use the canonical distance to the Crab nebula given by Davidson \& Fesen (1985), $d=1.83 \mathrm{kpc}$. The second term on the right-hand-side of Eq. (8) is the most important one - alas, it is also the most uncertain. Davidson \& Fesen (1985) give the estimate 2-3 $M_{\odot}$, while MacAlpine \& Uomoto (1991) evaluate this mass as 1-2 $M_{\odot}$. The most recent estimate was obtained by Fesen et al. (1997), who get much higher value $4.6 \pm 1.8 M_{\odot}$.
In what follows, we will consider two estimates of $M_{\text {neb }}$. The lower one, $2 M_{\odot}$, is a mean of the evaluations given by Davidson \& Fesen (1985) and MacAlpine \& Uomoto (1991). The higher one, $4.6 M_{\odot}$, uses the "central value" of the mass calculated by Fesen et al. (1997).

Most recent estimates of $n_{\mathrm{H}}$ are based on the evaluation of the hydrogen column density in the direction of Crab. Such a method yields $n_{\mathrm{H}} \simeq 0.5 \mathrm{~cm}^{-3}$ (Sollerman et al. 2000; Willingale et al. 2001). However, this might be a severe overestimate of $n_{\mathrm{H}}$, because the Crab radiation goes through the Galaxy disk on his way to the detector. In view of this, we will prefer to use a conservative value $n_{\mathrm{H}}=0.2 \mathrm{~cm}^{-3}$ quoted by Manchester \& Taylor (1977).

Using the above mentioned values of the quantities entering the right-hand-side of Eq. (8), we get two lower bounds for the moment of inertia $I_{\mathrm{Crab}}$, corresponding to two different estimations of the Crab nebula mass: $I_{\mathrm{Crab}, 45}>1.61$ for $M_{\text {neb }}=2 M_{\odot}$, and $I_{\mathrm{Crab}, 45}>3.04$ for $M_{\text {neb }}=4.6 M_{\odot}$.

\section{The $I(M, R)$ correlation}

In their seminal paper, Ravenhall \& Pethick (1994) studied the properties of the ratio $I / M R_{\infty}^{2}$, where $R_{\infty}$ stands for an apparent (radiation) stellar radius, determined in the analyzes of thermal radiation from the close by neutron stars,

$R_{\infty}=\frac{R}{\sqrt{1-2 G M / R c^{2}}}$

where $R$ is the coordinate radius of the space-time metric. In particular, they found that for an intermediate range of $\left(M / M_{\odot}\right)(\mathrm{km} / R) \sim 0.05-0.15$ the ratio $I / M R_{\infty}^{2}$ stays nearly constant, and depends rather weakly on the dense matter EOS. This finding resulted in their approximate formula for $I$.

In our quest for a universal, i.e., independent of the specific EOS approximate relation between $I, M$ and $R$, we relied on a large set of more than thirty EOSs of dense matter. This set includes various models of dense matter, which were obtained under different hypotheses on the composition of the matter at $\rho>2 \rho_{0}$, where $\rho_{0}=2.7 \times 10^{14} \mathrm{~g} \mathrm{~cm}^{-3}$ is the normal nuclear density. Considered compositions of the super-dense matter in neutron stars (NS) include: nucleons and leptons (EOSs of Baldo et al. 1997; Bombaci 1995; Balberg \& Gal 1997; Balberg et al. 1999; Bethe \& Johnson 1974; Pandharipande 1971; Pandharipande \& Ravenhall 1989; Douchin \& Haensel 2001; Wiringa et al. 1988; Walecka 1974; Haensel \& Prószyński 1980; Akmal et al. 1998); nucleons, hyperons, and leptons (EOSs of Glendenning 1985, 1997; Balberg \& Gal 1997; Weber et al. 1991; Balberg et al. 1999); deconfined quark matter mixed with baryonic matter (EOSs of Glendenning 1997); pion and kaon condensates coexisting with baryonic matter, in a pure or a mixed-phase configuration (EOSs of Glendenning 1997; Kubis 2001). We considered also three models of self-bound absolutely stable strange quark matter, forming hypothetical strange stars (SS; EOS of Haensel et al. 1986). After studying various types of correlations between $I, M$ and $R$, we found, in accordance with Lattimer \& Prakash (2001), that it is the ratio $I / M R^{2}$ which has the most 


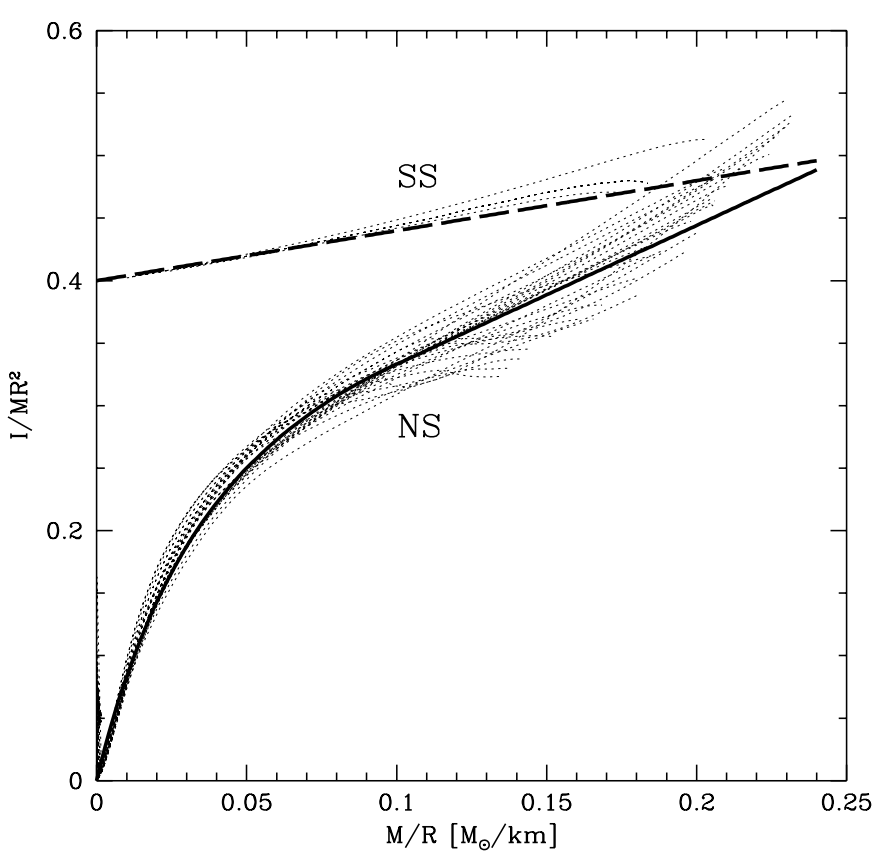

Fig. 1. The ratio $I / M R^{2}$ versus compactness parameter $M / R$ for selected set of EOSs of dense matter. Branches NS and SS correspond to neutron stars and strange stars. Thick lines were obtained using the analytical fitting formulae (solid - NS, dashed - SS), described in the text. Both thick lines are terminated at the $M / R$ upper-bound for the EOS respecting condition $v_{\text {sound }} \leq c$, which is equal 0.24 .

universal dependence on the compactness $M / R$. Our result is seen in Fig. 1.

In what follows, we will use, following Ravenhall \& Pethick (1994), the dimensionless compactness parameter $x \equiv$ $M / R \cdot \mathrm{km} / M_{\odot}$. The dependence of $I / M R^{2}$ on $x$ is well approximated, up to $M_{\max }$, by a function $a(x)$, different for neutron stars (NS) and strange stars (SS),

$a_{\mathrm{NS}}(x)= \begin{cases}x /(0.1+2 x), & \text { if } x \leq 0.1 \\ \frac{2}{9}(1+5 x), & \text { if } x>0.1\end{cases}$

$a_{\mathrm{SS}}(x)=\frac{2}{5}(1+x)$.

The difference in the $x$-dependence of $I / M R^{2}$ between neutron stars and strange stars stems from the qualitative difference in their density profiles. The density profile within a moderate mass strange star is very flat. On the contrary, density falls by many orders in the outer layer of a neutron star. Consequently, at a same $x \leq 0.2,\left(I / M R^{2}\right)_{\mathrm{SS}}>\left(I / M R^{2}\right)_{\mathrm{NS}}$ (the case of $x>0.2$ will be discussed later in this section). As we see in Fig. 1, the precision of the formulae given in Eq. (3) is typically better than $10 \%$. The formula for neutron stars breaks down only at very low $M$, below $0.2 M_{\odot}$. Such low masses do not seem relevant for neutron stars observed as pulsars ${ }^{1}$. The formula for

\footnotetext{
${ }^{1}$ Approximate formula for very low values of $M / R$ corresponding to the vicinity of the $M_{\min } \simeq 0.1 M_{\odot}$ is given in the footnote on p. 850 of Ravenhall \& Pethick (1994). (We believe, that we have found a couple of misprints in this footnote: the equation for very small $x=M / R$ should be rewritten as $I \simeq 7.5 \cdot M^{2} R$, and for greater $\left.x, I / M R^{2} \Lambda(R) \simeq 0.23-0.07 \cdot(1-10 x)^{2} \cdot \sqrt{x}\right)$.
}

$a_{\mathrm{SS}}$ looses its validity only for very low-mass $\left(M<0.1 M_{\odot}\right)$ strange stars with crust. On the contrary, the formula for $I$ becomes exact for low-mass bare strange stars, because they are Newtonian and their density is to a very good approximation constant, which corresponds to $a_{\mathrm{SS}} \simeq 2 / 5$.

The overall upper-bound on $x$ is reached at $M_{\max }$ for a pure causality-limit EOS $P=c^{2}\left(\rho-\rho_{\mathrm{s}}\right)$, and does not depend on $\rho_{\mathrm{s}}: x_{\max }^{\mathrm{CL}}=0.240$ (see Lindblom 1984; Haensel et al. 1999). Within General Relativity, static compact star models with EOS satisfying $v_{\text {sound }} \leq c$ have $x \leq x_{\max }^{\mathrm{CL}}$. We obtain $a_{\mathrm{NS}}\left(x_{\max }^{\mathrm{CL}}\right)=$ 0.489 and $a_{\mathrm{SS}}\left(x_{\max }^{\mathrm{CL}}\right)=0.496$, so that in the $x=x_{\max }^{\mathrm{CL}}$ limit $a_{\mathrm{SS}} \simeq a_{\mathrm{NS}}$ within about $1 \%$.

\section{Estimate of $I_{\mathrm{Crab}}$ and constraints in the $M-R$ plane}

In Sect. 2 we obtained two lower bounds for the Crab pulsar moment of inertia $I_{\text {Crab }}$, which depend on the different evaluations of the expanding shell mass: $I_{\text {Crab } 45}>1.61$ for $M_{\text {neb }}=2 M_{\odot}$ (conservative estimate), and $I_{\text {Crab,45 }}>3.04$ for $M_{\text {neb }}=4.6 M_{\odot}$ (newest estimate).

These bounds on $I_{\mathrm{Crab}}$, combined with our fitting formulae, derived in Sect. 3, imply constraints on the mass and radius of the Crab pulsar (for details, see Fig. 2). Neutron star configurations below the thick solid lines are ruled out. The weaker, conservative constraint eliminates a number of soft and moderately stiff EOS.

For those EOS which are acceptable, this constraint gives the range of possible mass and radius of the Crab pulsar, $M_{\mathrm{Crab}}>1.2 M_{\odot}$ and $R_{\mathrm{Crab}}=10-14 \mathrm{~km}$. The second $\left(M_{\text {neb }}=\right.$ 4.6 $M_{\odot}$ ) constraint is much more dramatic. Only a very stiff EOSs are accepted, because $M_{\text {Crab }}=1.9 M_{\odot}$ and $R_{\text {Crab }}=$ 14-15 km.

For the strange-star model we obtain strong constraints (thick dashed line in Fig. 2) even for the conservative estimate $M_{\text {neb }}=2 M_{\odot}: M_{\mathrm{Crab}}^{(\mathrm{SS})}>1.5 M_{\odot}, R_{\mathrm{Crab}}^{(\mathrm{SS})}=10-11 \mathrm{~km}$. Putting $M_{\text {neb }}=4.6 M_{\odot}$ excludes definitely the strange-star model. Note, however, that the strange-star constraint is likely to be an academic one anyway because the glitches in the timing of the Crab pulsar are widely believed to rule-out the strangestar model (Alpar 1987, nevertheless see Glendenning \& Weber 1992).

The formula expressing $I$ in terms of $M$ and $R$ may be useful for putting constraints, resulting for pulsar observations, on the neutron-star models. Consider a pulsar-powered supernova remnant of the Crab type. If the measured total power needed to sustain the radiation of the system and the acceleration of the nebula expansion is $\dot{E}_{\text {tot }}$, this implies the lower bound on the pulsar moment of inertia, $I>\dot{E}_{\text {tot }} /(\Omega|\dot{\Omega}|)$, where $\Omega$ and $\dot{\Omega}$ are measured pulsar angular frequency and its time derivative. Reliable estimates of $\dot{E}_{\text {tot }}$, combined with "empirical formula" for $I$, would then imply constraints on the neutron-star models in the $M-R$ plane. Bounds for the mass and radius of the Crab pulsar obtained for $M_{\text {neb }}=4.6 M_{\odot}$ are very strong and should therefore be considered with caution. More advanced models of the structure and evolution of the Crab nebula deserve to be developed. This problem will be addressed in a subsequent paper. 


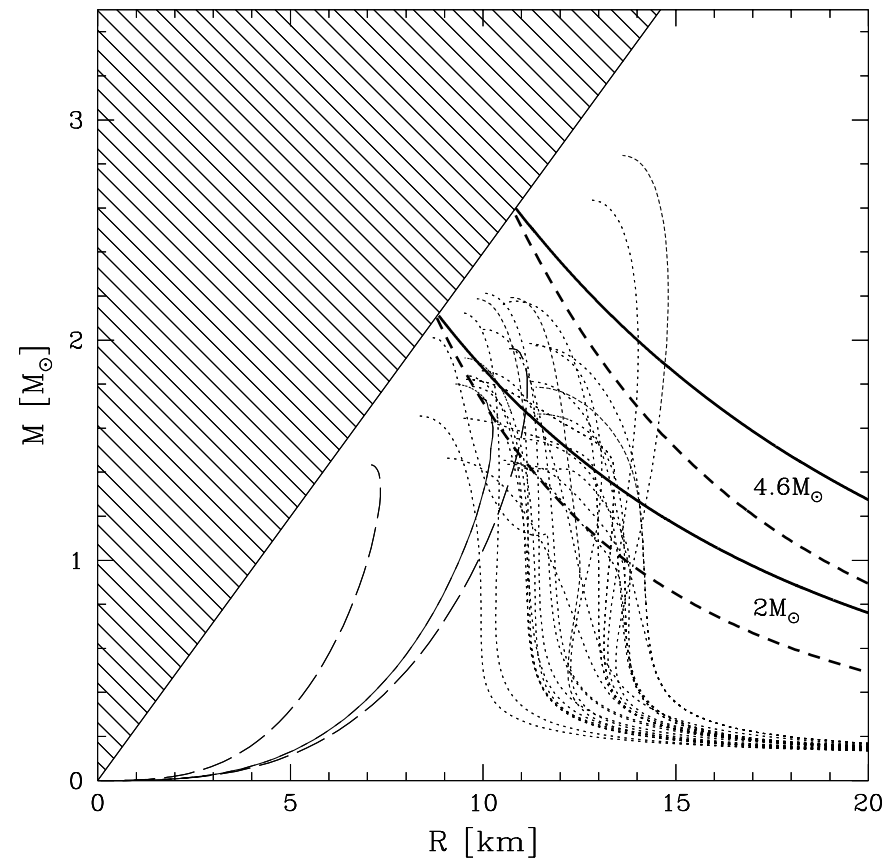

Fig. 2. The mass-radius curves for a large sample of EOS (thin dotted curves - neutron stars, thin dashed curves - strange stars) and constraints resulting from the estimates of $I$ of the Crab pulsar (thick solid line - neutron stars, thick dashed line - strange stars), obtained in the text. Hatched area is excluded by the General Relativity combined with $v_{\text {sound }} \leq c$.

Acknowledgements. We would like to thank Prof. B. Paczyński for helpful remarks. This research was partially supported by the KBN grant No. 5P03D.020.20.

\section{Appendix: Correlation between $I_{\max }$ and $M_{\max }$, $\boldsymbol{R}_{M_{\max }}$}

The fact that $a_{\mathrm{NS}}$ and $a_{\mathrm{SS}}$ converge at the highest values of compactness parameter suggests that there might exist a universal correlation between the maximum value of $I, I_{\max }$, and $M_{\max }$ and $R_{M_{\max }}$, valid for both neutron stars and strange stars. Such a correlation has been pointed out by Haensel (1990) for a set of nineteen EOS developed in 1970s and 1980s: $I_{\max , 45}=$ $0.98 \cdot\left(M_{\max } / M_{\odot}\right)\left(R_{M_{\max }} / 10 \mathrm{~km}\right)^{2}$. For the EOS set considered by Haensel (1990) this "empirical formula" reproduced values of $I_{\max }$ with an overall accuracy $\chi^{2}=0.03$. ${ }^{2}$ Within our sample of numerical results, obtained for selected thirty EOSs, we found correlation $I_{\max } \simeq C\left(M_{\max } / M_{\odot}\right)\left(R_{M_{\max }} / 10 \mathrm{~km}\right)^{2}$, the same as in (Haensel 1990), but with a slightly lower numerical factor $C=0.97$; the overall fit is characterized by $\chi^{2}=0.05$. The value of $\chi^{2}$ can be significantly reduced if one takes into account an additional correlation between the ratio

${ }^{2}$ In the present paper the value of $\chi^{2}$ is actually scaled by the variance, i.e., it is equal to the standard value of $\chi^{2}$ divided by $\sigma^{2}$. Such a treatment is convenient in our case, where we have to compare the qualities of different fits.
$I_{\max } /\left(M_{\max } R_{M_{\max }}^{2}\right)$ and the maximum compactness parameter $x_{\max }$ reached at $M_{\max }$. The improved empirical formula yields:

$I_{\max , 45} \simeq\left(-0.368+7.122 \cdot x_{\max }\right)\left(\frac{M_{\max }}{M_{\odot}}\right)\left(\frac{R_{M_{\max }}}{10 \mathrm{~km}}\right)^{2}$.

It is valid both for neutron stars and strange stars, with the overall quality of the fit $\chi^{2}=0.02$. The coefficient $C=0.97$ in the simplest empirical formula corresponds to an "average value of $x_{\max }$ ", $\bar{x}_{\max }=0.19$. Some general statements concerning different classes of the EOS can be made. The "empirical formula", Eq. (11), under-estimates $I_{\max }$ for the superluminal EOSs. On the contrary, it overestimates $I_{\max }$ for the EOSs with high-density softening due to the appearance of hyperons. Finally, for the "minimal composition EOSs" (nucleons and leptons) as well as for the strange matter EOSs, our formula reproduces $I_{\max }$ within better than $5 \%$.

A different, very precise formula for $I_{\max }$ of strange stars (bare or with crust) results from "scaling properties" (Haensel et al. 1999) of the configurations with $M_{\max }$ and $I_{\max }$. For the simplest model of strange quark matter composed of massless, non-interacting quarks, scaling with respect to the value of the bag constant implies exact relation $I_{\max , 45}=$ $0.94\left(M_{\max } / M_{\odot}\right)\left(R_{M_{\max }} / 10 \mathrm{~km}\right)^{2}$. After inclusion of the QCD interaction and finite strange-quark mass the above equation is no longer exact. For $m_{\mathrm{s}} c^{2}=(150-200) \mathrm{MeV}$, the numerical prefactor to be put in the expression for $I_{\max , 45}$ is 0.97 , and the formula is precise within a few percent.

One might argue that the validity of a simplest "empirical formulae" for $I_{\max }$ is a consequence of the fact that realistic EOSs are well approximated by the polytropes. For a polytropic EOS $P=K n_{\mathrm{b}}^{\Gamma}$ (where $n_{\mathrm{b}}$ is baryon density of matter) with a fixed $\Gamma$, the relation $I_{\max , 45}=C \cdot\left(M / M_{\odot}\right)\left(R_{M_{\max }} / 10 \mathrm{~km}\right)^{2}$ is exact, with $C$ independent of $K$. We get $C_{\Gamma=2}=0.83$ and $C_{\Gamma=3}=1.04$, so that the "best fit" value for realistic EOSs lies roughly in the middle of the $\Gamma=3$ and $\Gamma=2$ values. However, the realistic EOSs at super-nuclear densities are not polytropes. One can speak only about a local, density dependent $\Gamma\left(n_{\mathrm{b}}\right)$. For example, in the case of dense matter with hyperons the EOS softens considerably (and in a discontinuous way) at the thresholds for the appearance of a new hyperon, with $\Gamma$ dropping below one (Balberg \& Gal 1997). Clearly, the validity of the empirical formula for $I_{\max }$ is not due to the polytropic character of the EOS of dense matter.

\section{References}

Akmal, A., Pandharipande, V. R., \& Ravenhall, D. G. 1998, Phys. Rev. C, 58, 1804

Alpar, M. A. 1987, Phys. Rev. Lett., 58, 2152

Balberg, S., \& Gal, A. 1997, Nucl. Phys. A, 625, 435

Balberg, S., Lichtenstadt, I., \& Cook, G. B. 1999, ApJS, 121, 515

Baldo, M., Bombaci, I., \& Burgio, G. F. 1997, A\&A, 328, 274

Bethe, H. A., \& Johnson, M. B. 1974, Nucl. Phys. A, 230, 1

Bietenholz, M. F., Kronberg, P. P., Hogg, D. E., et al. 1991, ApJ, 373, L59

Bombaci, I. 1995, in Perspectives on Theoretical Nuclear Physics, ed. I. Bombaci, A. Bonaccorso, A. Fabrocini, et al., 223

Davidson, K., \& Fesen, R. A. 1985, ARA\&A, 23, 119

Douchin, F., \& Haensel, P. 2001, A\&A, 380, 151 
Douvion, T., Lagage, P. O., Cesarski, C. J., \& Dwek, E. 2001, A\&A, 373,281

Fesen, R. A., Shull, J. M., \& Hurford, A. P. 1997, AJ, 113, 354

Glendenning, N. K., \& Weber, F. 1992, ApJ, 400, 647

Glendenning, N. K. 1985, ApJ, 293, 470

Glendenning, N. K. 1997, Compact Stars: Nuclear Physics, Particle Physics and General Relativity (Springer, New York)

Haensel, P. 1990, in The magnetospheric structure and emission mechanisms of radio pulsars, ed. T. H. Hankins, J. M. Rankin, J. Gil (Pedagogical University Press, Zielona Góra, Poland), IAU Colloq. 128, 127

Haensel, P., Kutschera, M., \& Prószyński, M. 1980, A\&A, 102, 299

Haensel, P., Zdunik, J. L., \& Schaeffer, R. 1986, A\&A, 160, 121

Haensel, P., Lasota, J. P., \& Zdunik, J. L. 1999, A\&A, 344, 151

Kubis, S. 2001, Ph.D. Thesis, Jagellonian University (unpublished)

Lattimer, J. M., \& Prakash, M. 2001, ApJ, 550, 426

Lindblom, 1. 1984, ApJ, 278, 364

Lyne, A. G., \& Graham-Smith, F. 1998, Pulsar Astronomy (Cambridge Univ. Press)
MacAlpine, G. M., \& Uomoto, A. 1991, AJ, 102, 218

Manchester, R. N., \& Taylor, J. H. 1977, Pulsars (W. H. Freeman and Company, San Francisco)

Nugent, R. L. 1998, PASP, 110, 831

Pandharipande, V. R. 1971, Nucl. Phys. A, 147, 641

Pandharipande, V. R., \& Ravenhall, D. G. 1989, in Proc. NATO Advanced Research Workshop on nuclear matter and heavy ion collisions, Les Houches, 1989, ed. M. Soyeur, H. Flocard, B. Tamain, \& M. Porneuf (Plenum, New York, 1989), 103

Petersen, L. E. 1998, Rev. Mex. Astron. Astrofis. (Ser. Conf.), 7, 81

Ravenhall, D. G., \& Pethick, C. J. 1994, ApJ, 424, 846

Sollerman, J., Lundqvist, P., Lindler, D., et al. 2000, ApJ, 537, 861

Trimble, V. L. 1968, AJ, 73, 535

Weber, F., Glendenning, N. K., \& Weigel, M. K. 1991, ApJ, 373, 579

Willingale, R., Aschenbach, B., Griffiths, R. G., et al. 2001, A\&A, 365,212

Wiringa, R. B., Fiks, V., \& Fabrocini, A. 1988, Phys. Rev. C, 38, 1010 